\title{
Author Correction: Strong correlations and orbital texture in single-layer 1T- $\mathrm{TaSe}_{2}$
}

Yi Chen (D), Wei Ruan, Meng Wu, Shujie Tang (1), Hyejin Ryu, Hsin-Zon Tsai, Ryan L. Lee, Salman Kahn, Franklin Liou, Caihong Jia, Oliver R. Albertini, Hongyu Xiong (D), Tao Jia, Zhi Liu (D), Jonathan A. Sobota (1D, Amy Y. Liu, Joel E. Moore,

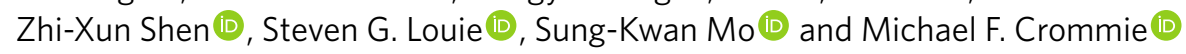

Correction to: Nature Physics https://doi.org/10.1038/s41567-019-0744-9, published 6 January 2020.

In the version of this Article previously published, co-author Ryan L. Lee was missing the middle initial. This has now been corrected in the online versions.

Published online: 3 June 2021

https://doi.org/10.1038/s41567-021-01283-3

(c) The Author(s), under exclusive licence to Springer Nature Limited 2021

\section{Publisher Correction: Ultracold gases: Second sound seen}

Sandro Stringari

Correction to: Nature Physics https://doi.org/10.1038/s41567-021-01276-2, published online 9 June 2021.

In the version of this News \& Views article originally published online, in ref. 1, the URL was missing the text '10.1038/' and should have read 'https://doi.org/10.1038/s41586-021-03537-9'. All versions of the article have been amended.

Published online: 14 June 2021

https://doi.org/10.1038/s41567-021-01297-X

(c) Springer Nature Limited 2021

\section{Addendum: Topological superconductivity: Quantized, finally}

\author{
Marcel Franz and Dmitry I. Pikulin
}

Addendum to: Nature Physics https://doi.org/10.1038/s41567-018-0100-5, published online 6 April 2018.

The Nature Article on which this News \& Views article is based was retracted on 8 March 2021 (see H. Zhang et al. Retraction Note: Quantized Majorana conductance. Nature 591, E30; 2021). Accordingly, any reference in the News \& Views article to the Zhang et al. paper and the results therein, in particular the reported conductance quantization, should be disregarded. That said, the discussion in the News \& Views article about what Majorana zero modes are and how quantized conductance might be an important piece of evidence for their existence remains correct.

Published online: 14 May 2021

https://doi.org/10.1038/s41567-021-01261-9

() Springer Nature Limited 2021 